\title{
A case study on displacement analysis of Vasa warship
}

https://doi.org/10.1515/jogs-2018-0006

Received November 11, 2017; accepted February 26, 2018

\begin{abstract}
Monitoring deformation of man-made structures is very important to prevent them from a risk of collapse and save lives. Such a process is also used for monitoring change in historical objects, which are deforming continuously with time. An example of this is the Vasa warship, which was under water for about 300 years. The ship was raised from the bottom of the sea and is kept in the Vasa museum in Stockholm. A geodetic network with points on the museum building and the ship's body has been established and measured for 12 years for monitoring the ship's deformation. The coordinate time series of each point on the ship and their uncertainties have been estimated epoch-wisely. In this paper, our goal is to statistically analyse the ship's hull movements. By fitting a quadratic polynomial to the coordinate time series of each point of the hull, its acceleration and velocity are estimated. In addition, their significance is tested by comparing them with their respective estimated errors after the fitting. Our numerical investigations show that the backside of the ship, having highest elevation and slope, has moved vertically faster than the other places by a velocity and an acceleration of about $2 \mathrm{~mm} /$ year and $0.1 \mathrm{~mm} /$ year $^{2}$, respectively and this part of the ship is the weakest with a higher risk of collapse. The central parts of the ship are more stable as the ship hull is almost vertical and closer to the floor. Generally, the hull is moving towards its port and downwards
\end{abstract}

Keywords: error estimation, coordinate and displacement time series, significance test

\footnotetext{
*Corresponding Author: Mehdi Eshagh: Department of Engineering Science, University West, Trollhättan, Sweden, E-mail: mehdi.eshagh@hv.se

Filippa Johansson, Lenita Karlsson: Department of Engineering Science, University West, Trollhättan, Sweden

Milan Horemuz: Division of Geodesy and Satellite Positioning, Royal Institute of Technology (KTH), Stockholm, Sweden
}

\section{Introduction}

Deformation monitoring of man-made structures, e.g. bridges, towers and dams, is one of the core tasks of geodesists. This is an important issue as such movements put lives at risk (Savvaidis 2003, Schroedel 2002). Therefore, knowing that constructions and buildings remain stable without of any risk of collapse is of tremendous significance. Monitoring the deformation of historical objects is important as they carry culture and thoughts to future generations. So they should be kept in good condition and in their original form and shape. By studying the movements of such objects over time, we can reconstruct, repair, strengthen, and preserve them for the future. In this paper, displacements of one of these historical objects in Sweden, the Vasa warship, are analysed statistically and the strong and weak points of this object are determined and visualised.

Deformation monitoring can be done by a variety of methods; by traditional surveying, Global Navigation Satellite Systems (GNSS), photogrammetric and remote sensing approaches or laser scanning. In the traditional method, the classical measurements like distance, angles and height differences are used to compute coordinates of different parts of a deforming object. Obviously geometric configuration of points constructing a network has a direct influence in the quality of coordinates being estimated. This is the reason why there are lots of studies about optimal design of classical networks (see e.g. Kuang 1996, Eshagh and Kiamehr 2007, Eshagh and Alizadeh-Khameneh, 2015a,b and Alizadeh-Khameneh et al. 2015).

Numerous studies have been done in deformation monitoring. Chen (1983) presented a generalised method for analysing deformation, Setan and Singh (2001) performed least-squares adjustment of a geodetic network and estimated time series of coordinate changes and modelled their trends to how each point on the deforming object moves. Hill and Sippel (2002) recommended combing geotechnical, motorised surveying instruments as well as GNSS sensors for deformation monitoring. Radovanovic and Teskey (2001) studied Global Positioning System (GPS) and the robotic tacheometric system for 
this purpose. Meng (2002) studied the real-time deformation of dams using GPS and accelerometer data. Cosser et al. (2003) took advantage of GPS, accelerometers, pseudolite and total stations for monitoring the deformation of a bridge. Dermanis and Kotsakis (2005) reviewed different methods for deformation monitoring. Significance of deformation was studies by Sanso and Calar de Lacy (2005) and Albertella et al. (2005). Integration of local and global frames without transformation for deformation monitoring purpose was investigated by Zurutuza and Sevilla (2005). The uncertainty of local geodetic deformation networks was studied by Chieca et al. (2005). Lima et al. (2005) compared the classical geodetic network and GPS with submillimetre accuracy over a large dam. Erol et al. (2005) used the GPS and levelling data for analysing deformation of a bridge to enhance the quality of vertical deformation measurements. Subsidence detection by GPS was performed by Esquivel et al. (2005) in Aguascalientes and they mentioned that the subsidence is about $18 \mathrm{~cm}$ at some points due to groundwater extraction and landslides. Raziq and Collier (2007) studied the GPS deflection monitoring of the West Gate Bridge in Melbourne and concluded that for engineering structures that exhibit smaller movements due to their stiffness GPS is not suitable. Andersson (2008) used the undifferenced GPS measurements to detect the displacement over Lilla Edet city in Sweden. Kaloop and Li (2009) used GPS for deformation monitoring of a bridge in China using Kalman filter and least-squares methods. Beshr and Kaloop (2013) used total station measurements to study and analyse deformation of another bridge using auto-correction adjustment techniques. Bagherbandi (2016) studied different adjustment methods for deformation monitoring based on total station observations. Amiri-Seemkooei et al. (2017) presented a method for analysing the stable points in a monitoring network. Niemeier and Tengen (2017) recommended a method for uncertainty assessment according to the guidelines for the expression of uncertainty in measurements and Monte-Carlo simulation.

The Swedish warship Vasa (Figure 1a) sank in 1628 on its maiden voyage, just 1000 metres after leaving port (http://www.vasamuseet.se/en/vasa-history/disaster/). It was salvaged in 1961 and is currently stored and displayed in the Vasa museum in Stockholm. During its 333 years at the bottom of the Baltic Sea decomposition and erosion of the ship occured. Most of the iron that was holding the ship together had rusted away, some of the wooden spars were eroded by the streams and sediment that the stream brought (http://www.vasamuseet.se/en/ vasa-history/salvage). The Vasa warship has been, and still is, studied carefully to monitor and detect all move- ments and changes in the body of the ship. Since October 2000, the displacement of this warship is monitored by Horemuz (2003). The data have been processed and analysed by Rosewarne (2007) as well. Over the hull there are 227 monitoring points, which can give a much clearer picture of how the ship is deforming due to the ongoing decomposition. This study is a continuation of the work done by Horemuz (2003) and Rosewarne (2007). Similar to the work of Setan and Singh (2001), we establish time-series of the coordinates and estimate the velocities and accelerations of all points and analyse them statistically according to the presented errors of the coordinates. The difference between our study and those done by Horemuz (2003) and Rosewarne (2007) is that we have modelled the velocity and acceleration of all points on the ship's hull and performed statistical analysis of them as well as the estimated displacements.

\section{Basic definitions of displacement, velocity and acceleration}

In surveying, displacement is known as coordinate change. It means that the coordinates of a point are measured repeatedly to see whether that point has moved or not. The displacement will have the same dimension as the coordinates, e.g. it can be detected in $x$-, $y$ - and $z$ dimensions at any point.

In order to detect displacements, some points on a deforming object are chosen and their coordinates are estimated repeatedly with respect to an $a$ priori defined reference frame. The coordinate difference of a point between each two successive epochs can be a measure for displacement. The displacement is a vector and it has both direction and magnitude (Croft et al. 2008, p. 628):

$$
\mathbf{d}=\mathbf{p}_{2}-\mathbf{p}_{1}
$$

where $\mathbf{d}$ is the displacement vector, $\mathbf{p}_{2}$ is the final and $\mathbf{p}_{1}$ the initial position vectors, respectively. Velocity $\mathbf{v}$, or displacement rate, is defined as the speed of displacement, which is obtained by dividing $\mathbf{d}$ by the time interval between the epochs:

$$
\mathbf{v}=\frac{\mathbf{d}}{t_{2}-t_{1}} \quad t_{2}>t_{1}
$$

where $t_{2}$ and $t_{1}$ are the times at which the coordinates are computed. The sign of velocity represents in which direction the object or point moves. Similarly, acceleration a is 
defined as rate of velocity:

$$
\mathbf{a}=\frac{\mathbf{v}}{t_{2}-t_{1}} \text { and } t_{2}>t_{1}
$$

If acceleration of a point has the same sign as its velocity, the point is accelerating, otherwise decelerating (Avison 1989, p.128).

As Eqs. (1a)-(1c) show, displacement, relative velocity and acceleration vectors are estimated once position of a point at two different epochs are measured. In the following, we explain the general idea of determining a displacement vector.

\section{Geodetic approach to displacement measurement}

Geodetic measurements consist of angles (horizontal and vertical), lengths and height differences, defined as polar coordinates, which need to be transformed into the Cartesian coordinates of $x^{-}, y-$, and $z$. For establishing a deformation monitoring network, the precision and accuracy of the measurements are of great importance. We always try to measure more quantities than the minimum necessary for coordinate estimation, this means that the number of measurements is larger than number of unknowns or coordinates. In such a situation, least-squares is the method to use for coordinate estimation. However, the mathematical models relating measurements and coordinates are normally nonlinear and need to be linearised. Such linearised formulae are written in a matrix form and solved in a leastsquares sense. In the following, this principle is briefly presented. For solving linearised equations approximate coordinates of the points are required $\left(\mathbf{x}^{0}\right)$, which can simply be estimated by solving a simple traverse connecting the points. $\mathbf{x}^{0}$ is used to reconstruct the measurements $\mathbf{L}^{0}$ and compare them with the real ones $(\mathbf{L})$. The difference between $\mathbf{L}$ and $\mathbf{L}^{0}$ denoted by $\Delta \mathbf{L}^{0}$ shows how far the coordinates are from what they should be. Therefore, a vector of corrections to the approximate coordinates $\left(\Delta \mathbf{x}^{1}\right)$ is estimated from $\Delta \mathbf{L}^{0}$ using $\mathbf{A}^{0}$ matrix, which is in fact a transformation matrix between $\Delta \mathbf{L}^{0}$ and $\Delta \mathbf{x}^{0}$ coming from the linearised mathematical models.

Suppose that our linearised system of equations forms a Gauss-Markov model:

$$
\mathbf{A}^{0} \Delta \mathbf{x}^{0}=\Delta \mathbf{L}^{0}-\boldsymbol{\varepsilon}, \quad \mathrm{E}\{\boldsymbol{\varepsilon}\}=0, \quad \mathrm{E}\left\{\boldsymbol{\varepsilon} \boldsymbol{\varepsilon}^{\mathrm{T}}\right\}=\sigma_{0}^{2} \mathbf{Q}
$$

$\Delta \mathbf{L}^{0}$ is an $n \times 1$ vector of differences between the real measurements and those estimated from the approximate coordinates, $\mathbf{A}^{0}$ is an $n \times m$ coefficients derived after lin- earisation of the mathematical model connecting the measurements and coordinates, $\Delta \mathbf{x}^{0}$ is the $m \times 1$ vector of corrections to the approximate coordinates and $\boldsymbol{\varepsilon}$ is an $n \times 1$ vector of random errors. $E\{$. $\}$ describes the statistical expectation operator. $\mathbf{Q}$ is the co-factor matrix of the observations and $\sigma_{0}^{2}$ is the a priori variance factor.

The least-squares solution of the Gauss-Markov model (3a) is:

$$
\Delta \hat{\mathbf{x}}^{0}=\left(\left(\mathbf{A}^{0}\right)^{\mathrm{T}} \mathbf{Q}^{-1}\left(\mathbf{A}^{0}\right)\right)^{-1}\left(\mathbf{A}^{0}\right)^{\mathrm{T}} \mathbf{Q}^{-1} \Delta \mathbf{L}^{0} .
$$

By adding the estimated corrections $\Delta \hat{\mathbf{x}}^{0}$ to $\mathbf{x}^{0}$, the approximate coordinates are updated. Thereafter, the coefficient matrix and measurements are reconstructed by the updated coordinates and the least-squares process is repeated for the new corrections and so on. This process is done until the corrections become significantly small or in other words, the solution has converged. The last update of the coefficients matrix (A) is used to estimate the variancecovariance matrix of the estimated coordinates:

$$
\hat{\mathbf{C}}_{\hat{\mathbf{x}}}=\hat{\sigma}_{0}^{2}\left(\mathbf{A}^{\mathrm{T}} \mathbf{Q}^{-1} \mathbf{A}\right)^{-1} \text { where } \hat{\sigma}_{0}^{2}=\frac{\hat{\boldsymbol{\varepsilon}}^{\mathrm{T}} \mathbf{Q}^{-1} \hat{\boldsymbol{\varepsilon}}}{n-m}
$$

and the residual vector will be:

$$
\hat{\boldsymbol{\varepsilon}}=\Delta \mathbf{L}-\mathbf{A} \Delta \hat{\mathbf{x}}
$$

where $\Delta \mathbf{L}$ is the last updated differences between the constructed measurements and real ones, and $\Delta \hat{\mathbf{x}}$ the last updated corrections.

Estimation of coordinates and their variancecovariance matrix should be repeated for every epoch of measurement so that by comparing the coordinates at two successive epochs, the coordinate change is seen. Let us consider two subsequent epochs of $i$ and $j$. The difference between two vectors of coordinates is nothing else than the displacement vector:

$$
\mathbf{d}_{i j}=\hat{\mathbf{x}}_{j}-\hat{\mathbf{x}}_{i}
$$

According to the error propagation law the variancecovariance matrix of the displacement vector is:

$$
\hat{\mathbf{C}}_{\mathbf{d}_{i j}}=\hat{\mathbf{C}}_{\hat{\mathbf{x}}_{j}}+\hat{\mathbf{C}}_{\hat{\mathbf{x}}_{i}} \text {. }
$$

Eq. (4a) contains displacement values of all points and Eq. (4b) the variance-covariance matrix of them. This means that, for example, the first 3 elements of $\mathbf{d}_{i j}$ are the displacement of a point in $x, y$ and $z$-directions and the first 3 elements of diagonal elements of $\hat{\mathbf{C}}_{\Delta \hat{\mathbf{x}}_{i j}}$ are the variances of the displacement in the corresponding directions and so on. 
For a single point the coordinates at epoch $i$ are $\mathbf{p}_{i}=$ $\left[\begin{array}{lll}\hat{x}_{i} & \hat{y}_{i} & \hat{z}_{i}\end{array}\right]^{\mathrm{T}}$ and at epoch $j \mathbf{p}_{j}=\left[\begin{array}{lll}\hat{x}_{j} & \hat{y}_{j} & \hat{z}_{j}\end{array}\right]^{\mathrm{T}}$, respectively. The displacement vector at this point will be:

$$
\mathbf{d}_{i j}=\mathbf{p}_{j}-\mathbf{p}_{i}=\left[\begin{array}{c}
\hat{x}_{j}-\hat{x}_{i} \\
\hat{y}_{j}-\hat{y}_{i} \\
\hat{z}_{j}-\hat{z}_{i}
\end{array}\right]=\left[\begin{array}{c}
\Delta \hat{x}_{i j} \\
\Delta \hat{y}_{i j} \\
\Delta \hat{z}_{i j}
\end{array}\right]
$$

and subsequently the error of displacement vector:

$$
\sigma_{\mathbf{d}_{i j}}^{2}=\left[\begin{array}{c}
\sigma_{\hat{\chi}_{j}}^{2}+\sigma_{\hat{\chi}_{i}}^{2} \\
\sigma_{\hat{y}_{j}}^{2}+\sigma_{\hat{y}_{i}}^{2} \\
\sigma_{\hat{z}_{j}}^{2}+\sigma_{\hat{z}_{i}}^{2}
\end{array}\right] .
$$

In order to see if the elements of $\mathbf{d}_{i j}$ are significant, their absolute values should be compared to their corresponding errors. If one element is smaller than its error, the displacement is not significant in a significance level of $68 \%$. Mathematically, this idea can be shown by:

$$
\begin{aligned}
& \left|\Delta \hat{x}_{i j}\right|>\sqrt{\sigma_{\hat{x}_{j}}^{2}+\sigma_{\hat{x}_{i}}^{2}} \\
& \left|\Delta \hat{y}_{i j}\right|>\sqrt{\sigma_{\hat{y}_{j}}^{2}+\sigma_{\hat{y}_{i}}^{2}} \\
& \left|\Delta \hat{z}_{i j}\right|>\sqrt{\sigma_{\hat{z}_{j}}^{2}+\sigma_{\hat{z}_{i}}^{2}}
\end{aligned}
$$

Similarly, the magnitude of the displacement vector can also be used. In this case, all three elements of position vectors are considered together and not only in the $x-, y$ - or $z$-direction. The 3D-displacement magnitude is:

$$
d=\left\|\mathbf{p}_{j}-\mathbf{p}_{i}\right\|=\sqrt{\left(x_{j}-x_{i}\right)^{2}+\left(y_{j}-y_{i}\right)^{2}+\left(z_{j}-z_{i}\right)^{2}} .
$$

As observed, Eq. (5a) is nonlinear with respect to the coordinates. In order to see if the magnitude of displacement $d$ is significant, its errors should be estimated and compared with $d$. The covariances between the coordinates of each point are available after performing the least-squares estimation and can be used to estimate the error of $d$ as well. For a point at two epochs of $i$ and $j$, the variance-covariance matrix of the coordinates is:

$$
\mathbf{C}_{i, j}=\left[\begin{array}{cccccc}
\sigma_{x_{i}}^{2} & \sigma_{x_{i} y_{i}} & \sigma_{x_{i} z_{i}} & 0 & 0 & 0 \\
\sigma_{x_{i} y_{i}} & \sigma_{y_{i}}^{2} & \sigma_{y_{i} z_{i}} & 0 & 0 & 0 \\
\sigma_{x_{i} z_{i}} & \sigma_{y_{i} z_{i}} & \sigma_{z_{i}}^{2} & 0 & 0 & 0 \\
0 & 0 & 0 & \sigma_{x_{j}}^{2} & \sigma_{x_{j} y_{j}} & \sigma_{x_{j} z_{j}} \\
0 & 0 & 0 & \sigma_{x_{j} y_{j}} & \sigma_{y_{j}}^{2} & \sigma_{y_{j} z_{j}} \\
0 & 0 & 0 & \sigma_{x_{j} z_{j}} & \sigma_{y_{j} z_{j}} & \sigma_{z_{j}}^{2}
\end{array}\right]
$$

where $\sigma_{x_{i}}^{2}, \sigma_{y_{i}}^{2}$ and $\sigma_{z_{i}}^{2}$ are, respectively, the variances of $x$-,y- and $z$-coordinate at epoch $i$, and $\sigma_{x_{j}}^{2}, \sigma_{y_{j}}^{2}$ and $\sigma_{z_{j}}^{2}$ are the corresponding ones at epoch j. $\sigma_{x_{i} y_{i}}$ stands for covariance between $x$ and $y$-coordinates, $\sigma_{x_{i} z_{i}}$ between $x$ - and $z$ coordinates and $\sigma_{y_{i} z_{i}} y$ and $z$ at epoch $i$ and $\sigma_{x_{i} y_{j}}, \sigma_{x_{j} z_{j}}$ and similarly $\sigma_{y_{j} z_{j}}$ covariances at epoch $j$. As observed, there is no covariance between the coordinates at two different epochs, this means that the measurements at one epoch are independent from another epoch.

By applying the error propagation law, the variance of $d$ will be:

$$
\sigma_{d}^{2}=\mathbf{B C}_{i, j} \mathbf{B}^{\mathrm{T}}
$$

where $\mathbf{B}$ is the coefficients matrix of the equation with the following structure:

$$
\mathbf{B}=\left[\begin{array}{llllll}
\frac{\partial d}{\partial x_{i}} & \frac{\partial d}{\partial y_{i}} & \frac{\partial d}{\partial z_{i}} & \frac{\partial d}{\partial x_{j}} & \frac{\partial d}{\partial y_{j}} & \frac{\partial d}{\partial z_{j}}
\end{array}\right],
$$

and the elements of $\mathbf{B}$ are partial derivatives of $d$ with respect to the coordinates at epochs $i$ and $j$. The magnitude of displacement will be considered significant if it is larger than its error, namely:

$$
d>\sigma_{d}
$$

So far, two methods for analysing the significance of displacements have been presented. The first one is applied to the displacement vector and shows that in which direction the displacement is significant. The second one is applied to the displacement magnitude.

\section{Equations of motion of hull points}

It is well-known in physics that the equation of motion of a moving object with a constant acceleration is derived by solving a second-order differential equation with respect to time:

$$
\frac{\mathrm{d}^{2} f(t)}{\mathrm{d} t^{2}}=a
$$

where $f(t)$ is any function of time. For example, in this study, $f$ can be $x, y$ or $z$-coordinate, and $a$ stands for the constant acceleration. Integrating Eq. (6a) twice with respect to time $t$ yields (see also Croft et al. 2008, p.182):

$$
f(t)=\frac{1}{2} a\left(t-t_{0}\right)^{2}+v\left(t-t_{0}\right)+f\left(t_{0}\right)
$$

and $t_{0}$ is the initial time and $v$ stands for velocity.

Eq (6b) is a quadratic polynomial, which can be fitted to any time series of coordinates for estimating the acceleration $a$, velocity $v$ and initial coordinate $f\left(t_{0}\right)$. Considering that values of coordinates $f(t)$ are available at different epochs, we can organise a system of equations of GaussMarkov type to solve the unknown parameters $a, v$, and 
$f\left(t_{0}\right)$. In this case, the matrix form of the system of equation will be:

$$
\mathbf{K u}=\mathbf{W}-\boldsymbol{\varepsilon}^{\prime} \text { and }, \mathrm{E}\left\{\boldsymbol{\varepsilon}^{\prime}\right\}=0, \mathrm{E}\left\{\boldsymbol{\varepsilon}^{\prime} \boldsymbol{\varepsilon}^{\prime \mathrm{T}}\right\}=\sigma_{0}^{2} \mathbf{Q}^{\prime}
$$

where $\boldsymbol{\epsilon}^{\prime}$ is the random error and

$$
\begin{aligned}
& \mathbf{K}=\left[\begin{array}{ccc}
\frac{1}{2}\left(t_{1}-t_{0}\right)^{2} & \left(t_{1}-t_{0}\right) & 1 \\
\frac{1}{2}\left(t_{2}-t_{0}\right)^{2} & \left(t_{2}-t_{0}\right) & 1 \\
\vdots & \vdots & \vdots \\
\frac{1}{2}\left(t_{n}-t_{0}\right)^{2} & \left(t_{n}-t_{0}\right) & 1
\end{array}\right], \mathbf{u}=\left[\begin{array}{l}
a \\
v \\
f\left(t_{0}\right)
\end{array}\right] \\
& \mathbf{W}=\left[\begin{array}{c}
f\left(t_{1}\right) \\
f\left(t_{2}\right) \\
\vdots \\
f\left(t_{n}\right)
\end{array}\right]
\end{aligned}
$$

Least-squares solution of Eq. (7a) is:

$$
\hat{\mathbf{u}}=\left(\mathbf{K}^{\mathrm{T}} \mathbf{Q}^{\prime-1} \mathbf{K}\right)^{-1} \mathbf{K}^{\mathrm{T}} \mathbf{Q}^{\prime-1} \mathbf{W} .
$$

The variance-covariance matrix of the estimated parameters will be:

$$
\hat{\mathbf{C}}_{\hat{\mathbf{u}}}=\frac{(\mathbf{K} \hat{\mathbf{u}}-\mathbf{W}) \mathbf{Q}^{\prime-1}(\mathbf{K} \hat{\mathbf{u}}-\mathbf{W})}{n-3}\left(\mathbf{K}^{\mathrm{T}} \mathbf{Q}^{\prime-1} \mathbf{K}\right)^{-1}
$$

where $n$ is the total number of epochs. The significance of the estimated acceleration and velocity can be tested according to their respective estimated errors.

\section{Numerical results}

The described ideas are now applied to the coordinate time series of 227 monitoring points at the Vasa warship hull. It is of importance to study how the displacements at these points behave to prevent the ship from the risk of collapse. The errors and covariances between the coordinates of each point are available and need to be considered in our analysis. However, coordinates at each epoch are independent from one another. In the following section, a general overview of the geodetic network of this ship is presented.

\subsection{Geodetic Network of Vasa warship}

The geodetic network consists of control, station and detail points - see Figure 2. There are 24 control points realised by prisms mounted on the concrete walls of the museum building, which define a local coordinate frame used for the deformation analysis. They are evenly distributed (in the horizontal and vertical direction) around the ship and together with 49 total station points they form a geometrically strong 3D control network. The coordinates of the total station points are not considered as fixed in the adjustment. These station points are located on five floors (five height levels). Three to five control and 10 - 20 detail points marked by reflective tapes on the hull are measured from each station in every measurement epoch. Each detail point is measured from at least two station points. So far 34-measurement epochs during 17 years were performed.

Since the ship is decomposing slowly, the displacements will be very small and therefore, the measurements' precision plays a significant role. The points are evenly distributed with a good geometry in the museum building and a local reference coordinate system was defined for describing the coordinates. After the least-squares solution of the coordinates, a standard error of $0.1 \mathrm{~mm}$ was achieved for the individual coordinates except for few points having precision of $0.2 \mathrm{~mm}$. For the first seven epochs of measurements the standard error of individual coordinates of the monitored points ranges between 0.1 and $0.7 \mathrm{~mm}$. The largest errors are related to those points on the ship's masts, but they are not used in this study, as the masts are not firmly attached to the ship.

The $x$-axis of the defined reference frame is along the port/starboard side of the ship, and $y$-axis along the stern/bow side and perpendicular to the $x$-axis. The $z$-axis is along the plumb line and upwards. The coordinates of the monitored points were computed by a constrained network adjustment - the coordinates of control points are fixed. Before this adjustment, the between-epoch stability of the control network was checked by a separate free network adjustment, where only observations towards the control points are included. The stability was also checked by a special measurement epoch in 2006, where the control points were measured with greater redundancy. No significant changes of the control network were detected. Two observation epochs have been performed every year: one in spring and one in fall, so the time interval between epochs is 6 months.

As mentioned before, there are 227 points on the ship's hull, but we select 16 of them, which shows the shape of the ship approximately. Figure $1 \mathrm{~b}$ shows the position of the selected points and the coordinate frame. However, we emphasise that we have analysed all 227 points on the deforming hull. As we can see, the ship is rather long with a length of about $50 \mathrm{~m}$, width of $10 \mathrm{~m}$ and a height of $15 \mathrm{~m}$. Each point has a unique code and 16 selected points are presented in Figure 1b with their codes. 3 points, which are highlighted by red circles, are those we have used for presenting the coordinate times series. Presenting the time 
series of all 16 points is not very conclusive and makes the paper long. Figure 1c shows the geodetic monitoring points around and on the hull of the ship. The red triangles are the control points, those blue circles stations points and the black dots the points on the ship's hull.

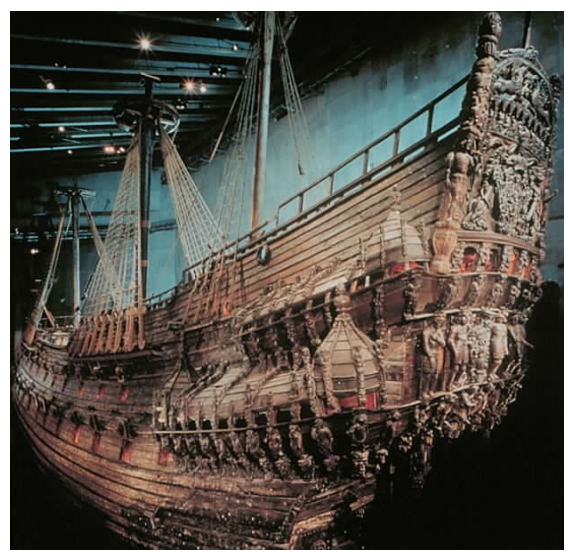

(a)

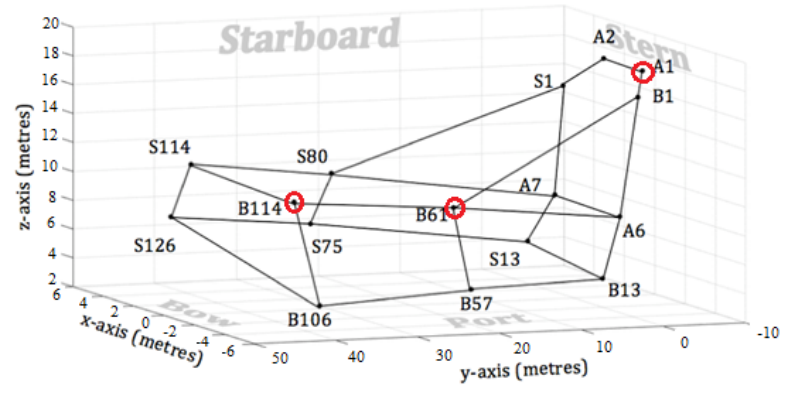

(b)

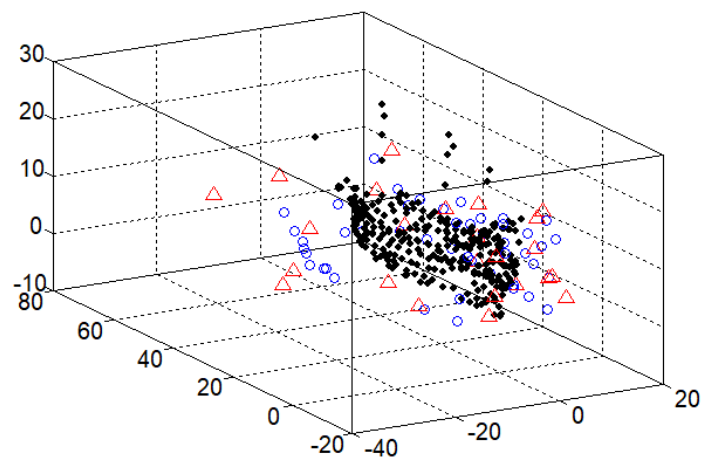

(c)

Figure 1: a) The photo of the ship in the VASA museum, b) 16 selected points over the ship's hull, c) Points of geodetic monitoring network. Red triangles - control points, blue circles - station points and black dots - detail points on the hull.

\subsection{Time series of coordinate changes}

Here, we plot the coordinate time series of three points of A1, B114 and B61 for visualisation purpose. What we can see in them is that the coordinates are mainly decreasing. For example, $\mathrm{A} 1$ is a decreasing point, meaning that its $x$-, $y$ and $z$-coordinates are decreasing. Since generally the equation of motion is a quadratic polynomial, we used Eq. (6b) and fitted it to each coordinate time series, to estimate its acceleration and velocity. The red line presented in Figure 12 is the plot of this polynomial. The $x$ - and $y$ coordinate time series of the point B114 is not very conclusive as we observe variations without a specific trend, but that of the $\mathrm{z}$-coordinate is decreasing. The coordinate time series of the point B61 can be interpreted and explained similarly. Generally, we can say the ship is becoming more stable and the changes in the $\mathrm{z}$-coordinates of the points are being decreasing.

The quadratic polynomial is fitted to all 227 points and the acceleration, velocity and the initial coordinate of each point have been estimated during 22 epochs to see which part of the ship moves faster than the other. The presented plots in Figure 1 show that the behaviour of the points is different depending on their position on the ship. Those points located at the stern are behaving in one way and the ones on the bow in another.

\subsection{Significance of the accelerations and velocities}

Here, the estimated accelerations and velocities as well as their errors for all coordinate times series on the ship's hull are plotted. The accelerations and velocities will be significant if their absolute values are larger than their corresponding errors. Figure 3 shows these absolute values and the errors. The horizontal axis of the plots is the number of points, and vertical axis the accelerations and velocities in the units of $\mathrm{mm} / \mathrm{year}^{2}$ and $\mathrm{mm} /$ year. The black plots show the absolute values of the estimated accelerations or velocities and the red ones their corresponding estimated errors.

Comparing the $x$-coordinate accelerations and their errors, presented in Figure 3a, we can see that there are points having larger error than the acceleration itself. Similarly, the velocities presented in Figure $3 \mathrm{~b}$ can be explained. Figure $3 \mathrm{c}$ is the plots of the accelerations along the $y$-axis and their errors. As we observe, they are all larger than the estimated errors and therefore significant. The same is true for the velocities presented in Figure 3d. Figure 3 e and 3 represent corresponding plots for the $z$-axis 


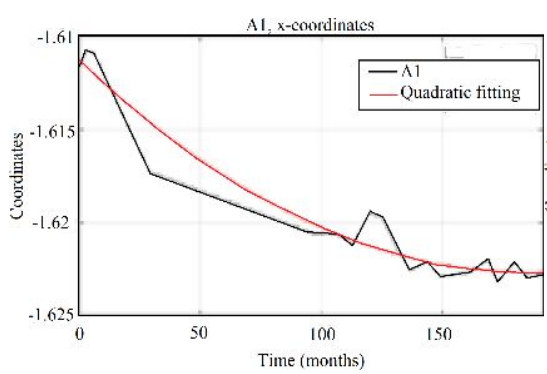

(a)

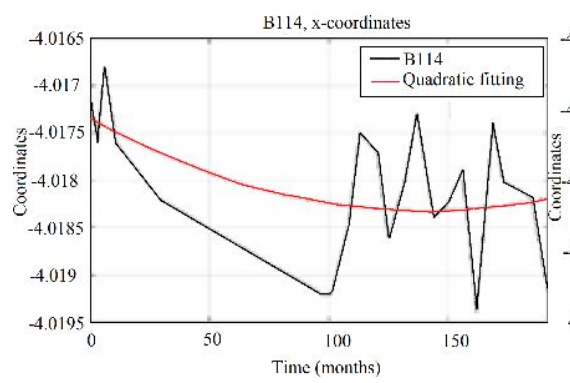

(d)

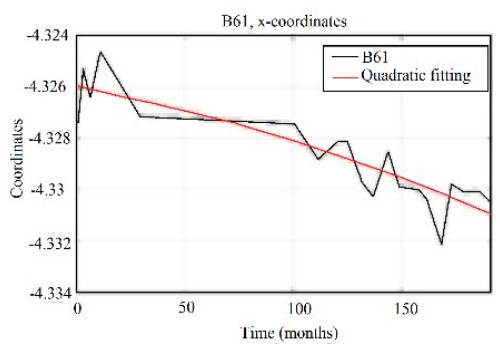

(g)

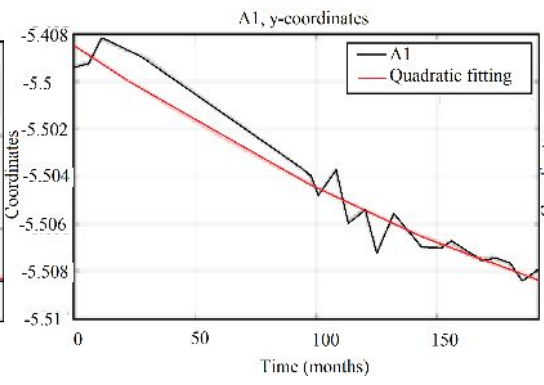

(b)

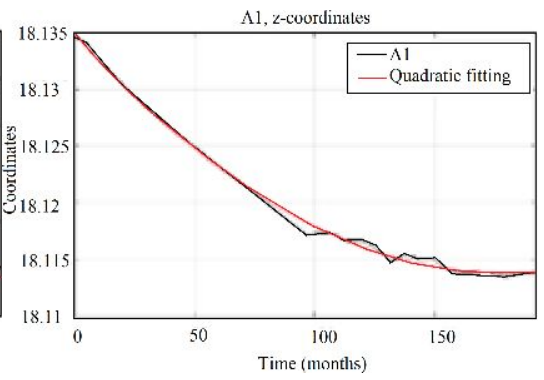

(c)

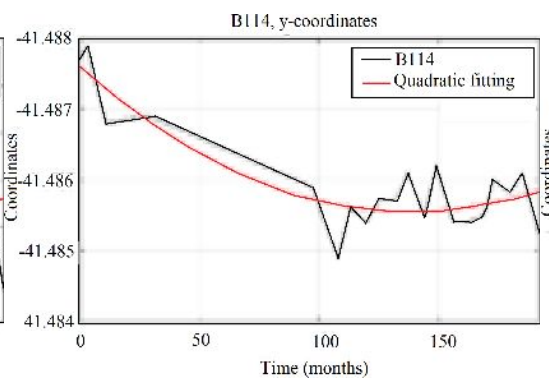

(e)

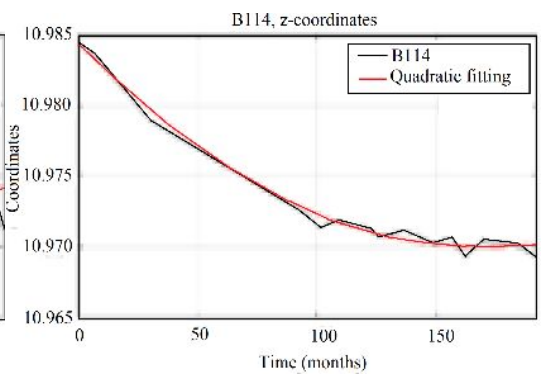

(f)

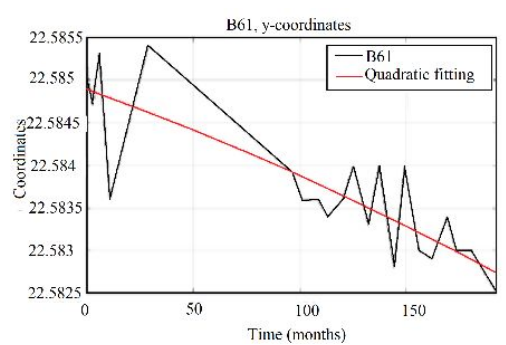

(h)

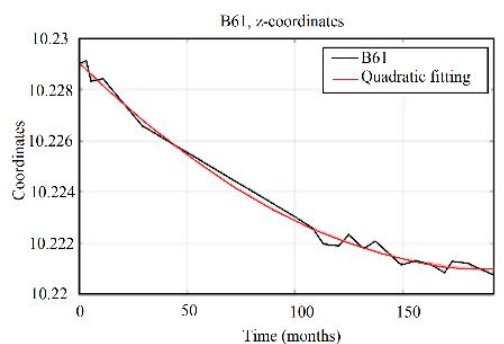

(i)

Figure 2: Time series of coordinates for points A1, B114 and B61.

and showing they are all significant. We have to mention that since the absolute values of the accelerations and velocities are considerably larger than the errors, plots of the errors look like a line with zero values in Figures 3c, d, e, and $f$.

Since we observe that there are some points with insignificant accelerations and velocities along the $x$-axis, we compute the ratio of all accelerations and velocities and their errors. Those points having ratios larger than 1 are considered as deforming points and those with smaller fixed ones. Figures $4 \mathrm{a}$ and $4 \mathrm{~b}$ show, respectively, the insignificant accelerations and velocities along the $x$-axis. As we observe these insignificant values are located in the lower part of the ship's hull and close to the ship's centre.

By fitting a quadratic polynomial to the coordinate time series, we could estimate the accelerations and velocities of all points in $x, y$, and $z$ directions. Also, we showed in Figure 3 that their significant values are along the $z$ - direction. Figures $5 \mathrm{a}$ and $5 \mathrm{~b}$ show them on the hull of the ship. It is clearly visible that the highest parts, or the stern side, have the largest values. This indicates that the ship is unstable in the vertical due to lack of any support in this area. The lowest accelerations and velocities are seen in the midsection, as this part is closest to or partially on the floor. Second highest accelerations and velocities are found along the $y$-axis, showing that the ship is tilting towards the starboard or portside.

\subsection{Significance of the displacements}

To test the statistical significance of displacements, they are compared with their standard deviations, errors. Those having larger errors than their own values had insignificant changes. Since there are 227 points on the ship measured 22 times, 21 values can be computed for each point. 


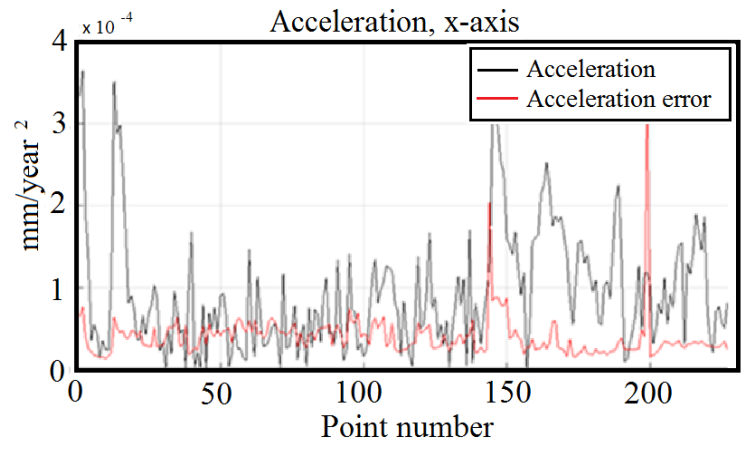

(a)

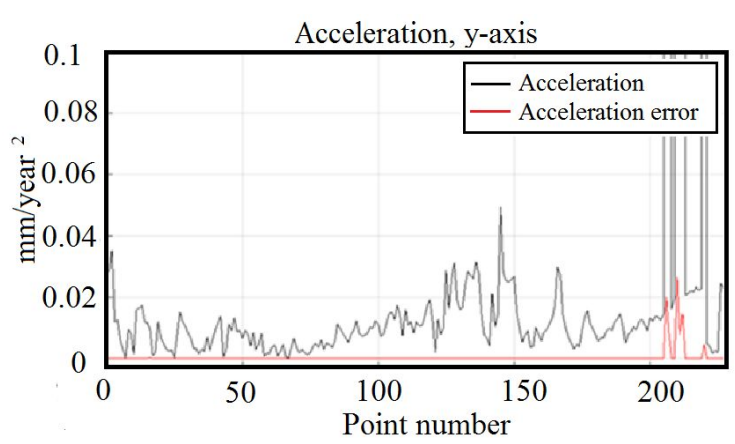

(c)

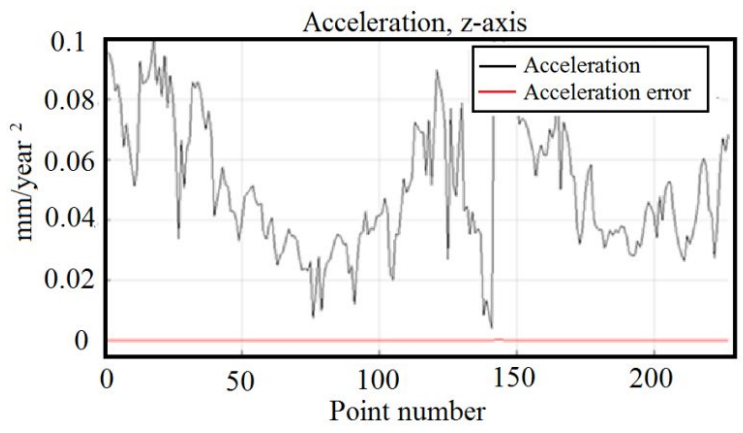

(e)

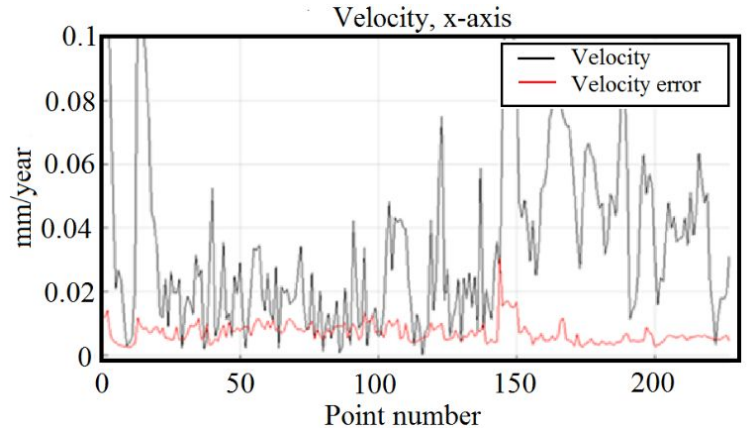

(b)

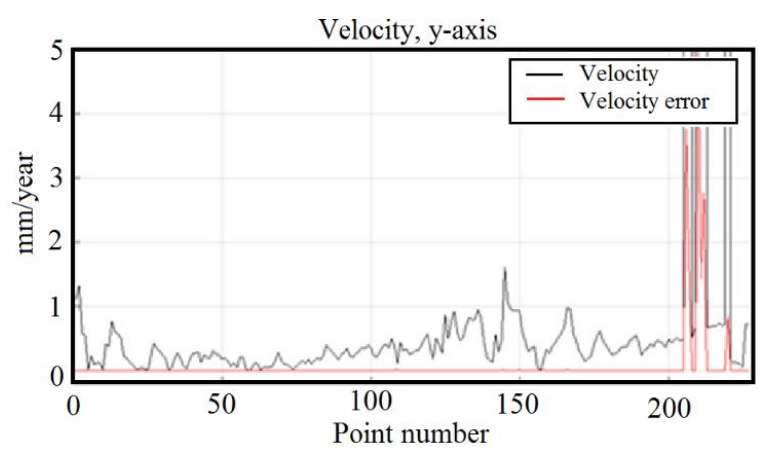

(d)

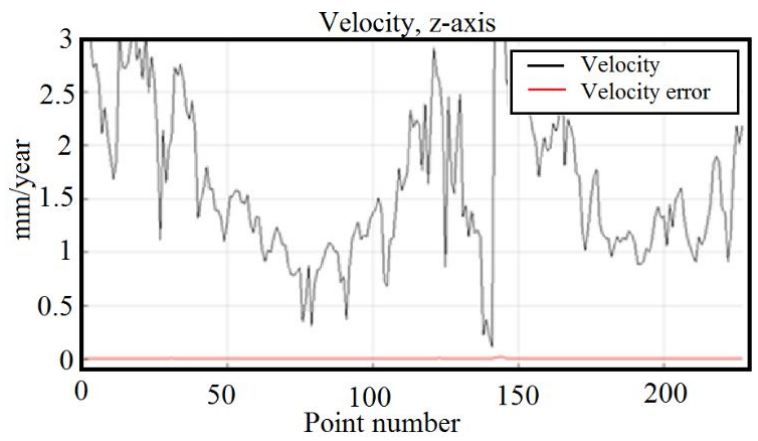

(f)

Figure 3: Plot of a) and b) $x$-coordinate accelerations and velocities and their errors of all points on the hull of the ship, c) and d) $y$ coordinate acceleration and velocity with their errors, e) and f) those of $z$-coordinate

Here, we just present the displacement and their errors only for the points A1, B61 and B114 in Figure 6.

The spike in the plots of the displacement is due to the time gap between the epochs 5 and 6 . Generally, the errors are smaller than the displacements at the point A1. The point B61 has small displacements but at the last epochs the displacements and errors are in the same order. A similar statement can be said for the point B114, but the errors are considerably larger at epochs 18 and 19.

For presenting Figure 6, no covariance between the coordinates of each point could be used. However, these covariances have been also computed during the least- squares solution of the coordinate and can be considered when computing the errors of the displacements. Here, we will show how much the computed displacement errors differ in between the cases of considering and ignoring the covariances. This investigation was performed for all points on the ship's hull, but here we present only the errors for those 3 selected points of A1, B61 and B114 in Figure 7. In this figure, the red line shows the errors without considering the covariances and the black one includes covariances. At the point A1, no significant change is seen in the estimated errors in both cases. However, for the points B61 and B114, a larger error is seen when the covariances 


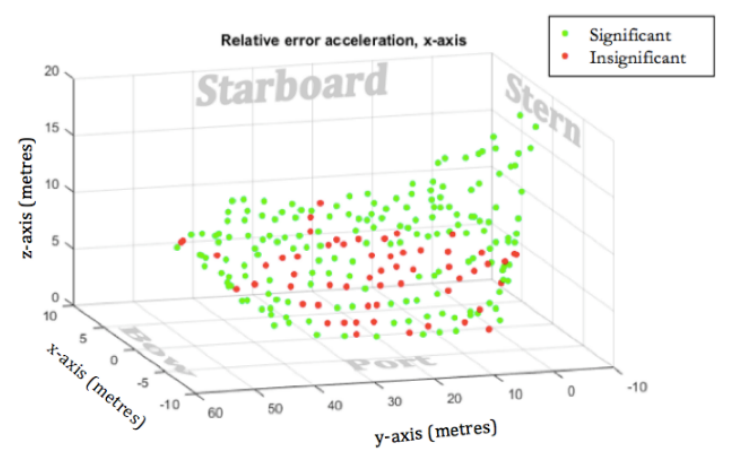

(a)

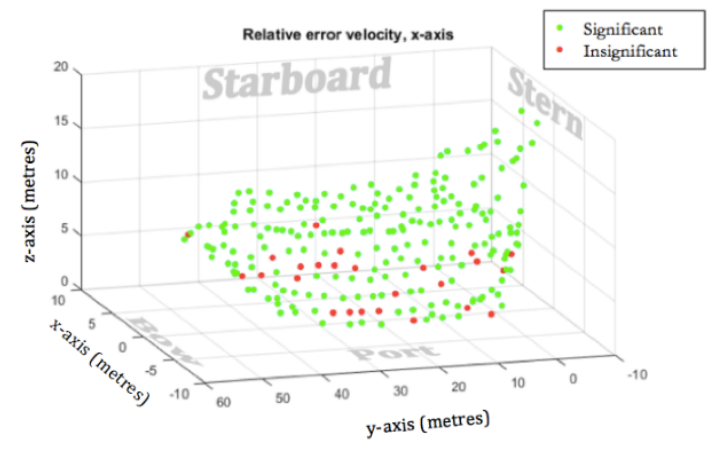

(b)

Figure 4: Significant and insignificant a) accelerations and b) velocities along the $x$-axis.

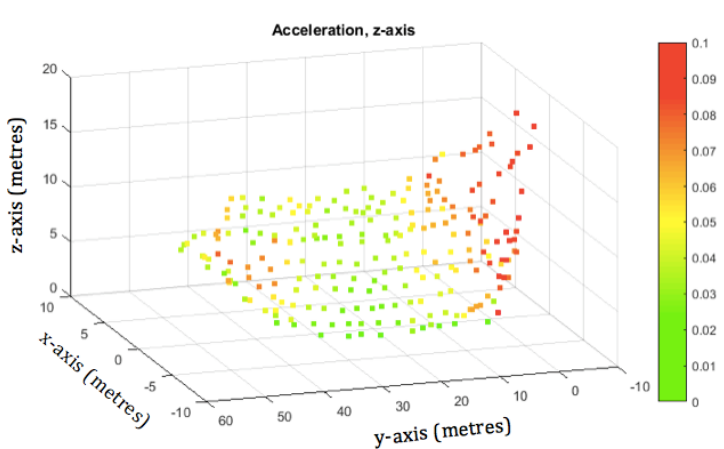

(a)

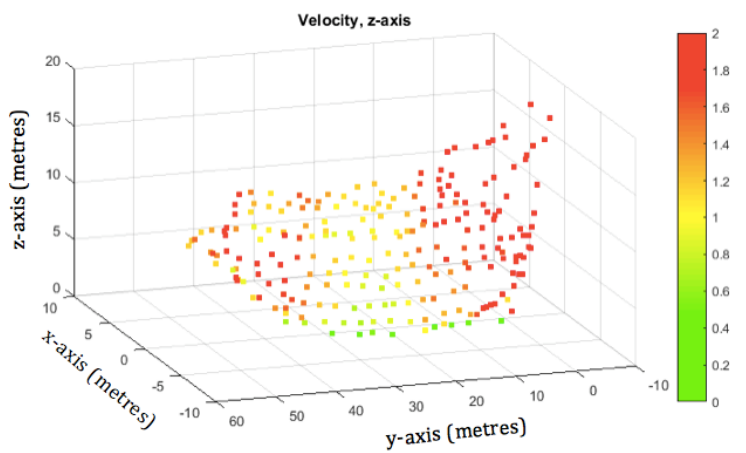

(b)

Figure 5: a) Accelerations [mm/year $\left.{ }^{2}\right]$ and b) velocities along $z$-axis [mm/year]

are considered. In any case, we observe the differences between these cases are not very significant as the errors are still at the same order and considerably smaller than the displacements. Note that the largest coordinate changes occur in the $z$-coordinates and the changes in $x$ and $y$ directions are not very significant comparing to that of $z$. Therefore, it is evident that the displacement along the $z$ direction will have the largest contribution to the displacement computed by Eq. (5a). So the small changes in the estimated errors due to involvement of covariance do not change the results comparing to the case where the covariances are ignored.

In Figure 8, we show the total displacements over the body of the ship. Displacements are multiplied by 50 and added to the initial coordinates at the selected points for the purposes of improved visualisation. As can be seen, all points have moved towards the ship's port while at the same time going downwards, in other words, the body of the ship is tilting clockwise.

\section{Conclusions}

Our numerical investigations confirm the previous studies and show that the ship is deforming downwards. It deforms faster at the stern and at the starboard side of the bow than other parts. The general reason is that the ship is decomposing and its strength is decreasing with time. Having a high vertical rate of change is explained by the attraction of gravity pulling down the ship continuously. The back and front sides suffer by decomposing due to carrying more weight, and the concave shape of the ship's hull. Those parts constructed with a slope are more influenced by gravity than the other sides and those having small displacements are almost constructed vertically. The estimated velocities reach to $2 \mathrm{~mm} /$ year and the accelerations to $0.1 \mathrm{~mm} /$ year2 at some points, especially those at the stern part. Therefore, the risk of collapse is higher for back end of the ship, which has higher elevation and 


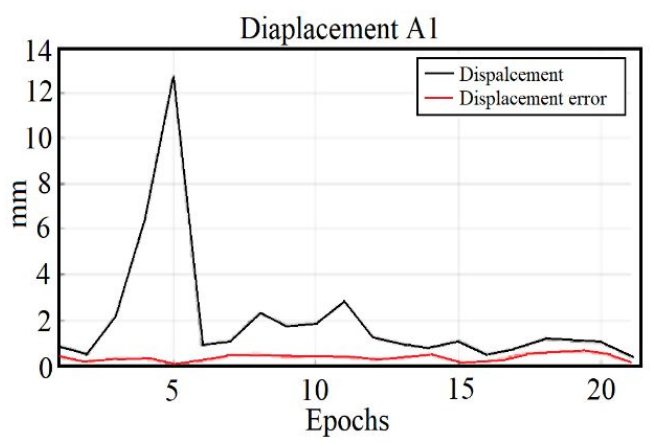

(a)

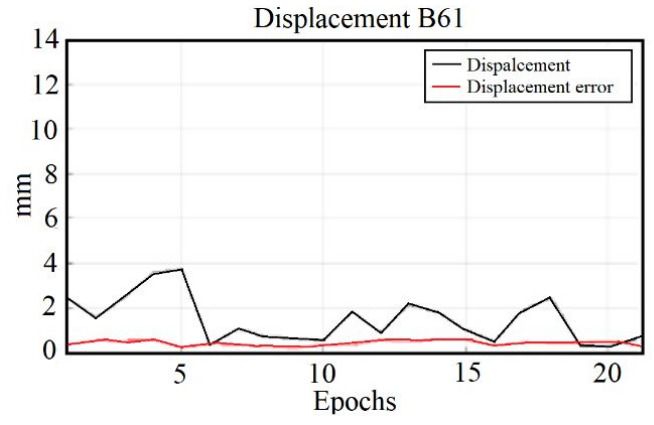

(b)

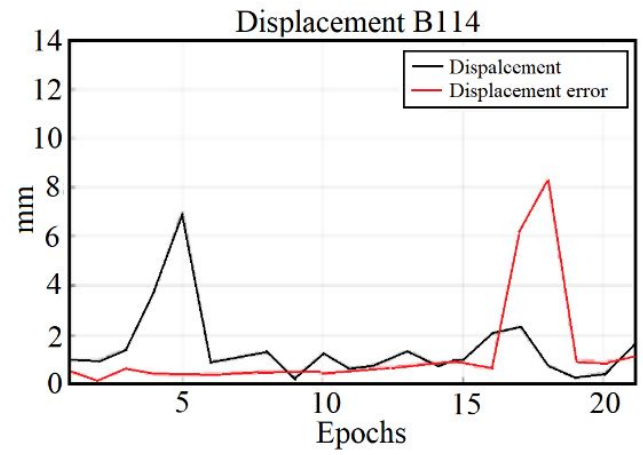

(c)

Figure 6: Plots of displacements and their errors for points a) A1, b) B161 and B114.

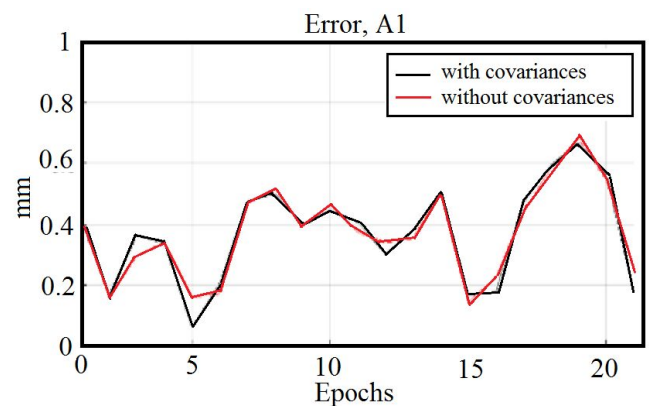

(a)

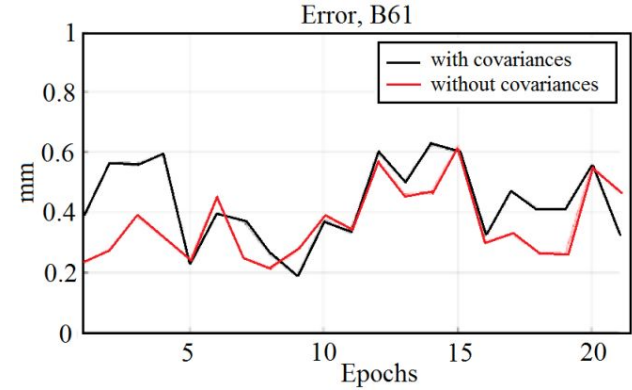

(b)

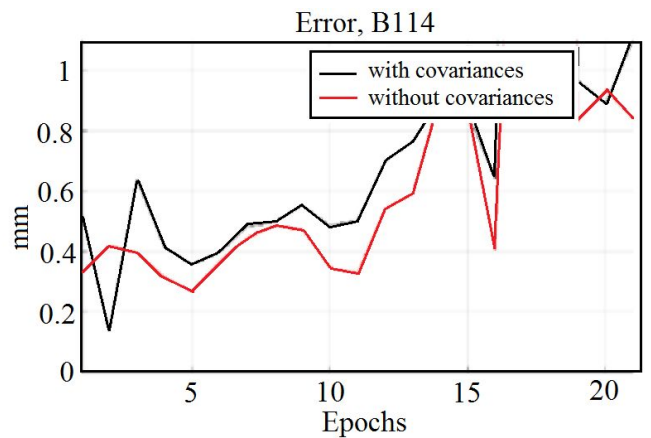

(c)

Figure 7: 3D-errors with and without covariances between coordinates at point a) A1, b) B61 and c) B114. 


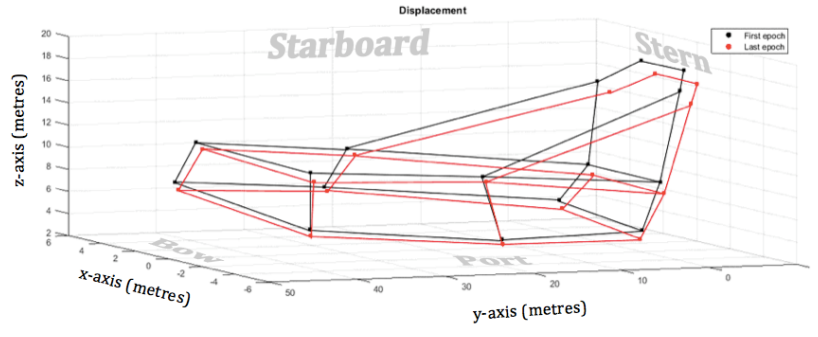

Figure 8: The magnified 3D-displacements (multiplied by 50).

slope. We should emphasise that we cannot predict the ship deformation in the future using these velocities and accelerations, as a quadratic polynomial has been fitted to the time series of the coordinates and in most of the cases the polynomial is concave meaning that if they are used for prediction in the future wrong results will be obtained. These values show that the ship has moved by these velocities and accelerations during these 22 epochs of measurement.

In this study, an alternative method was presented for analysis of displacements and their errors. In this method, estimated covariances between the coordinates of each point can also be considered in error estimation. This method was applied in two scenarios; considering and ignoring covariances. The conclusion was that the errors, in spite of being different in each scenario, have no significant effect in the analysis of displacements and what we conclude about the displacement significance in the case where covariances are ignored will be valid for the other scenario as well. Therefore, considering covariances is not important in this method of displacement analysis.

Acknowledgement: The Vasa Museum is cordially acknowledged for making available the necessary information and data for this study. Dr. Linn Gustavsson is appreciated for her encouragement to write this article.

\section{References}

Albertella A, Cazzania N., Sanso F., Sacerdote F., Crespi M. and Luzietti L. (2005) Deformation detection by a Bayesian approach prior information representation and testing criteria definition, In Geodetic Deformation Monitoring: from geophysical to engineering roles (Eds. Sanso F. and Gil A.J.), 131, 30-37.

Alizadeh-Khameneh, MA, Eshagh M. and Sjöberg L.E. (2015) Optimisation of Lilla Edet Landslide GPS Monitoring Network, Journal of Geodetic Science, 5:57-66.

Amiri-Simkooei A.R., Alaei-Tabatabaei S.M. and Zangeneh-Nejad F. (2017) Stability analysis of deformation-monitoring network points using simultaneous observation adjustment of two epochs,
Journal of Surveying Engineering, 143, 1,

Andersson J. V. (2008) A complete model for displacement monitoring based on undifferenced GPS observations, PhD thesis in Geodesy, Royal Institute of Technology (KTH), Sweden.

Avison J. (1989) The World of Physics, 2 ed. Cheltenham: Nelson Thornes Ltd.

Bagherbandi M. (2016) Deformation Monitoring using Different Least Squares Adjustment Methods: A Simulated Study, KSCE Journal of Civil Engineering (2016) 20(2):855-862

Beshr A. A. El.-W. and Kaloop M. R. (2013) Monitoring bridge deformation using auto-correlation adjustment technique for total station observations. Positioning, Vol. 4, No. 1, pp. 1-7,

Chen Y. Q. (1983) Analysis of deformation surveys - A generalized method, Technical Report No. 94, Department of Surveying Engineering, University of New Brunswick, Fredericton, N.B.

Chieca M., Baselga S., Berné J.L. and Maestro I.C. (2005) Uncertainty modelling in local geodetic networks, In Geodetic Deformation Monitoring: From geophysical to engineering roles (Eds. Sanso F. and Gil A.J.), 131, 56-62.

Cosser E., Roberts G. W., Xiaolin M. and Dodson A. H. (2003) Measuring the dynamic deformation of bridges using a total stations. Proceedings, 11th FIG Symposium on Deformation Measurements, Santorini, Greece.

Croft A. and Davison R. (2008) Mathematics for engineers: a modern interactive approach. 3. ed. Harlow: Pearson Education

Dermanis A and Kotsakis C. (2005) Estimating crustal deformation parameters from geodetic data: Review of existing methodologies, open problems and new challenges, In Geodetic Deformation Monitoring: grom geophysical to engineering roles (Eds. Sanso F. and Gil A.J.), 131, 7- 18.

Erol S., Erol B. and Ayan T. (2005) Analyzing the deformation of a bridge using GOS and levelling data, In Geodetic Deformation Monitoring: From geophysical to engineering roles (Eds. Sanso F. and Gil A.J.), 131, 244-253.

Eshagh M. and Alizadeh-Khameneh M.A. (2015) Two-epoch optimal design of displacement monitoring networks, Boletim de Ciências Geodésicas, 21,3, 484-497.

Eshagh M. and Kiamehr R. (2007) A strategy for optimum designing of the geodetic networks from the cost, reliability and precision views, Acta Geodetica et Geophysica Hungarica, 42, 2, 297-308.

Eshagh M. and Alizadeh K. M. A (2015) The effect of constraints on bi-objective optimization of geodetic networks, Acta Geodetica et Geophysica Hungarica, 50, 449-459.

Esquivel R., Hernandez A., Zermeno M.E. (2005) GPS for subsidence detection, the case study of Aguascalientes, In Geodetic Deformation Monitoring: From geophysical to engineering roles (Eds. Sanso F. and Gil A.J.), 131, 254- 258.

Hill, C. D. and Sippel, K. D. (2002) Modern deformation monitoring: a multi sensor approach, FIG XXII International Congress, Washington DC, USA.

Horemuz M. (2003) Deformationsmätningar av skeppet Vasa. SINUS, 3, 5-8.

Kaloop M. R. and Li H. (2009) Monitoring of bridge deformation using GPS technique. KSCE Journal of Civil Engineering, Vol. 13, Issue 6, pp. 423-431.

Kuang S. L. (1991) Optimization and design of deformation monitoring schemes, Technical Report No. 157. PhD Thesis, Department of Surveying Eng. University of New Brunswick, Canada.

Kuang S. L. (1996) Geodetic network analysis and optimum design: concepts and applications, Ann Arbor Press, Inc., Chelsea, Michi- 
gan.

Lima N., Casaca J. and Henriques M.J. (2005) Accuracy of displacement monitoring at large dams with GPS, In Geodetic Deformation Monitoring: From geophysical to engineering roles (Eds. Sanso F. and Gil A.J.), 131, 239-243.

Meng X. (2002). Real-time deformation monitoring of bridges using GPS/accelerometers, PhD Thesis, University of Nottingham, Nottingham, UK.

Niemeier W. and Tengen D. (2017) Uncertainty assessment in geodetic network adjustment by combining GUM and Monte-Carlosimulations, Journal of Applied Geodesy, 11, 2, 67-76.

Radovanovic R. S. and Teskey W. F. (2001) Dynamic monitoring of deforming structures: GPS verses robotic tacheometry systems. The 10th FIG International Symposium on Deformation Measurements, Orange, California, USA, pp. 61-70.

Raziq N. and Collier P. (2007) GPS deflection monitoring of the west gate bridge, 3rd IAG / 12th FIG Symposium, Baden, May 22-24.
Rosewarne S. (2007) Deformation study of the Vasa Ship. Master thesis, Royal Institute of Technology (KTH), Stockholm, Sweden.

Sanso F. and Carla de Lacy M. (2005) The Bayesian Approach applied to significance deformation identification, In Geodetic Deformation Monitoring: From geophysical to engineering roles (Eds. Sanso F. and Gil A.J.), 131, 19-29.

Savvaidis P.D. (2003) Existing Landslide Monitoring Systems and Techniques, In Proceedings of the conference from stars to earth and culture, In honor of the memory of Professor Alexandros Tsioumis, The Aristotle University of Thessaloniki. Thessaloniki, Greece, 242-258.

Setan H. and Singh R. (2001) Deformation analysis of a geodetic monitoring network. Geomatica, Vol. 55, No. 3.

Zurutuza J. and Sevilla M.J. (2005) Deformation monitoring by integrating local and global reference systems, In Geodetic Deformation Monitoring: From geophysical to engineering roles (Eds. Sanso F. and Gil A.J.), 131, 48-55. 